\title{
Level the Buffer Wall: Fair Channel Assignment in Wireless Sensor Networks
}

\author{
Yanyan Yang, Yunhuai Liu, and Lionel M. Ni \\ Department of Computer Science and Engineering, Hong Kong University of Science and Technology \\ yanyan,yunhuai@cse.ust.hk,ni@cs.ust.hk
}

\begin{abstract}
In this paper, we study the trade-off between network throughput and fairness in a multi-channel enabled wireless sensor network (WSN). Traditional approaches attempt to solve the two problems in an isolated manner without a joint design. Our empirical studies show that solutions to these two problems cannot be simply combined. Away from the traditional belief, the number of channels in WSNs with Telosb sensor nodes operating at $2.4 \mathrm{GHz}$ band can be up to 83 and the orthogonal channels can be up to 27 . The switching overhead in terms of time and energy cost is relatively small. Furthermore, we observe a buffer wall phenomenon which is one of the main reasons causing network throughput degradation and unfairness. To strike a better trade-off between the network throughput and fairness, we design a novel multi-channel assignment algorithm, targeting at maximizing the minimal data sending rate. The key idea of the proposed algorithm is to level down the buffer wall so that the buffer usage of nodes can be evenly distributed. As such, the bandwidth of bottleneck nodes can be fully utilized and the unfairness due to the node locality can be removed. We prove that the achieved data sending rate is no less than $4 / 9$ of the optimal rate in theory. Our experimental results show that the minimal data sending rate can be improved by up to $100 \%$ comparing with the existing work TMCP.
\end{abstract}

Key Words: WSN; Fairness; Buffer Wall Phenomenon; Multichannel Assignment;

\section{INTRODUCTION}

In a wireless sensor network (WSN) [1], sensor nodes are typically deployed in the sensing field to periodically report the sensed data to the sink. Such a many-to-one communication pattern, also known as data aggregation, is a common feature in many WSN-based applications (e.g., [2], [3]). Network throughput, which is defined as the amount of sensory data that can be successfully transmitted to and collected at the sink per unit of time, has been the primary metric to evaluate the performance of various data aggregation algorithms (e.g., [4], [5]). As sensor nodes are deployed to cover different regions in a sensing field, it is often essential to guarantee that sensory data from different regions of the sensing field can be fairly delivered. Thus, besides the network throughput, another important evaluation metric of data aggregation algorithms is fairness among sensor nodes in terms of their opportunity to deliver their own sensory data to the sink. A major objective of this research is trying to maximize the minimal achievable data sending rate generated from each individual sensor node.

One way to increase the network throughput in WSN is to take advantage of the availability of multiple channels. Though each sensor node is typically equipped with a single network interface and can operate in only one channel at a time, different nodes can be assigned to different channels so that the interference and collision can be largely reduced. Although much research has been dedicated to multi-channel assignment in wireless networks (e.g., [6], [7]), these works were, however, more theoretical than practical, without paying much attention to practical issues, such as how many channels are really available, what the channel switching time is, and what the energy cost is for channel switching. The lack of empirical studies greatly limits the application of these approaches in a real environment.

In the meanwhile, few works have been devoted to the fairness aspect. Though some researchers have studied the fairness issue in single channel environments (e.g., [8], [9]), to the best of our knowledge, none of them has considered the advantages of multi-channel availability. Moreover, a simple combination of the multi-channel assignment and fairness control cannot fully take the possible advantages. This is mainly due to the buffer wall phenomenon revealed by our empirical studies. When a packet, either carries its own sensory data or carries sensory data of others to be forwarded, cannot be transmitted by a sensor node due to channel unavailability or other reasons, the packet will be accumulated in the buffer for later delivery. Traditionally it is believed that the nodes nearer to the sink are more likely to be congested than the ones away from the sink (e.g., [10], [11]). The buffer wall phenomenon points out that in many cases, the nodes nearer to the sink are likely to have empty buffers due to the lack of data to transfer, while the buffers of the nodes that are further away to the sink are filled with data. Because of this observation, the major challenge to achieve greater fairness and higher throughput simultaneously is to level down the buffer wall to get an ideal buffer usage distribution.

To address this problem, we propose a new multi-channel assignment algorithm called double-plate. Double-plate is a layer-based approach which allows the nodes in the same layer of the same sub-routing tree to choose the same sending channel. The nodes work in an asynchronous slot based manner to reduce the channel switching overhead and to guarantee the successful transmission rate. In order to achieve fairness among different sub-routing trees, an inter sub-routing tree rate adaptation algorithm is also proposed. To sum up, the main contributions of this paper are as following:

- We show that the problem of multi-channel assignment on-demand of high network throughput and fairness cannot be easily overcome by a simple combination of 
existing solutions in the two domains.

- We conduct a series of empirical studies to investigate the characteristics of multi-channel in WSNs using a Telosb based test-bed. The experimental results show that there are more than 80 channels available, which is quite different from the previous belief. We also study the channel switching time and energy consumptions. These results are the basis of the channel assignment protocol.

- We study the buffer usage distribution of individual sensor nodes in WSNs. We give the fundamental observation of the existence of buffer wall phenomenon. We show that by leveling down the buffer wall, a higher per flow rate is expected by striking a better trade-off between throughput and fairness.

- We propose double-plate, a channel assignment protocol that fully uses the channels in a more beneficial way. Simulations and evaluations on Telosb nodes are made, indicating that at least a $4 / 9$ optimal data sending rate per node can be achieved with $100 \%$ goodput per node, which achieves $100 \%$ improvement comparing to the known TMCP [6].

The rest of the paper is organized as follows. Related work is illustrated in Section II. Section III shows the empirical results of the multi-channel availability and the existence of the buffer wall. In Section IV, a general channel assignment protocol is proposed with the detailed design addressing major challenges, such as intra sub-routing tree channel switching scheme and inter sub-routing tree data sending rate adaptation. Section V shows the simulation and evaluation results. At last, we conclude the paper in Section VI.

\section{RELATED WORKS}

The related work can be classified into multi-channel assignment protocols and fairness-oriented protocols. Most past research related to multi-channel assignment protocols were targeted for mobile ad hoc networks (MANETs) rather than for WSNs (e.g., SSCH [12], TMMAC [13]). The main objective of these protocols is to improve the network throughput. Furthermore, these protocols were designed for MANETs and do not work well in WSNs due to two major constraints. First, nodes in MANETs may have multiple full duplex radio interfaces, whereas sensor nodes in WSNs typically have one half duplex radio due to the cost and power constraints. Second, sensor nodes have very limited computational and storage capabilities. Thus, computation and communication intensive algorithms may not be appropriate for WSNs.

One of the most recent research for multi-channel assignment in WSNs is TMCP [6]. TMCP assigns each node a single fixed channel. Nodes using the same channel form a tree, which is actually a sub-routing tree rooted at the sink. Since TMCP focuses on providing a practical protocol to improve the network throughput, many issues are open if we take fairness into consideration. First, the nodes in the same sub-routing tree communicate in the same channel. They still suffer from the buffer wall problem and cannot achieve a higher data sending rate from each individual node. Second, TMCP claims that there is less benefit to assign one node with multiple channels as the number of channels is quite limited. Our empirical results, however, suggest that in the $2.4 \mathrm{G}$ band, there are a large number of channels available (see Section III.A). Such results provide the basis for our work that it is possible for one node to switch among multiple channels to level down the buffer wall.

Although many researchers have suggested to allow a node to switch among multiple channels, most of the work are theoretically interesting without much realistic experiment support (e.g., MMSN [7]). These works mainly assign channels in an interference-free manner. Each node is assigned with a fixed listening channel differing from other nodes. During operation, one node must firstly switch to its receiver's listening channel for data transmission, which introduces lots of extra energy cost for coordination communication.

Some researchers have paid attention to fairness-oriented protocol design in the case of a single channel, such as IFRC [8] and RCRT [9]. In their studies, nodes are supposed to generate packets in a common rate and buffer usage for individual nodes is the main criterion for rate adaptation. But the achievable data sending rate is far less than the optimal rate due to the buffer wall phenomenon. In order to get an ideal buffer usage distribution, besides taking the advantages of multi-channel availability, the distribution of buffer usage among all sensor nodes should be carefully considered.

\section{EMPIRICAL STUDIES}

In this section we show some empirical study results which are the main motivation for our work. We first investigate the characteristics in the use of multiple channels in WSNs, and then present the observations of the buffer wall phenomenon. Our experiments use Telosb sensor nodes which are equipped with the $\mathrm{CC} 2420$ radio device operating at the $2.4 \mathrm{G}$ openspectrum band. Each Telosb runs TinyOS and CSMA/CA is adopted as the MAC layer protocol.

\section{A. Multi-channel availability}

The CC2420 radio device is able to switch channels with the minimum interval of $1 \mathrm{MHz}$ [15] resulting in a total of 83 $1 \mathrm{MHz}$-away channels, from $2.4 \mathrm{G}$ to $2.4835 \mathrm{G}$. Our investigations include the transmission capacity of wireless channels, the interferences between different channels, and the switching overhead in terms of the time and energy consumption.

1) Transmission capacity of wireless channels: In the first set of experiments, a pair of nodes, one sender and one receiver, are involved. The sender keeps sending packets one after another with its highest capability. The receiver listens to the channel and records how many packets have been successfully received. Each packet has a fixed length of 43 bytes [14]. Fig. 1 plots the capacity in each of the 83 channels (Kbps) and Fig. 2 plots the corresponding successful transmission rate (STR). From these two figures we can find that there are no significant differences among different channels. The maximal rate is about $50.6 \mathrm{Kbps}$ and the minimal is $49.5 \mathrm{Kbps}$, with the difference no more than $2 \%$. The STR of different channels 


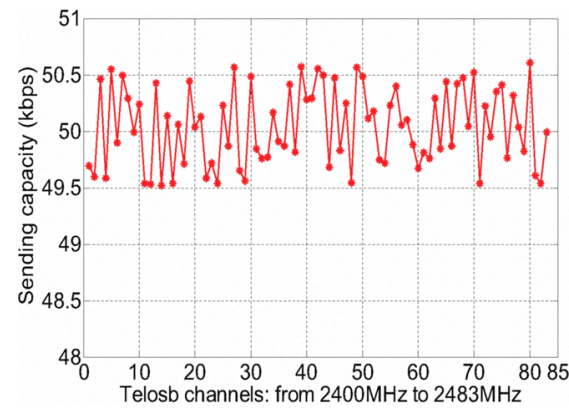

Fig. 1. Sending capacity for each $2.4 \mathrm{G}$ band channel on Telosb

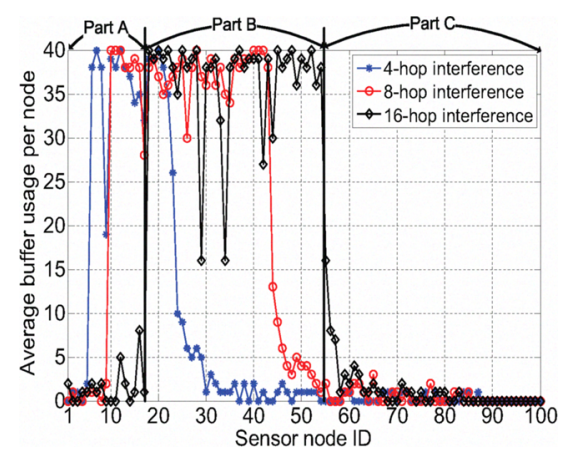

Fig. 4. Impact of interference range to the buffer wall phenomenon

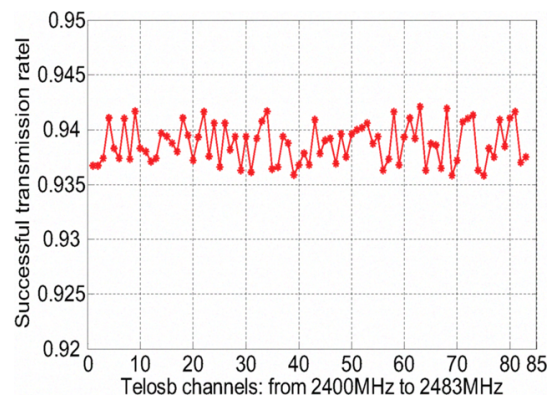

Fig. 2. Successful transmission rate for each 2.4G band channel on Telosb

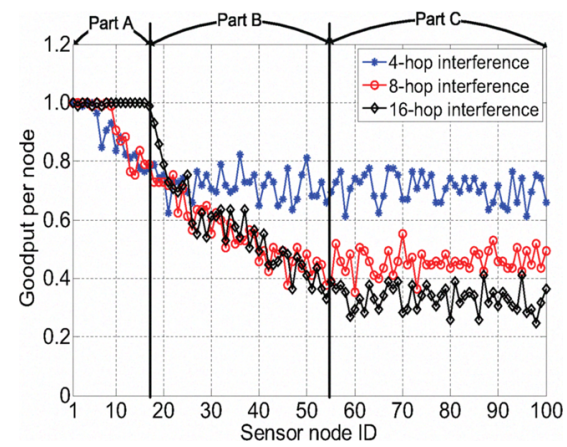

Fig. 5. Impact of interference range to the goodput per node

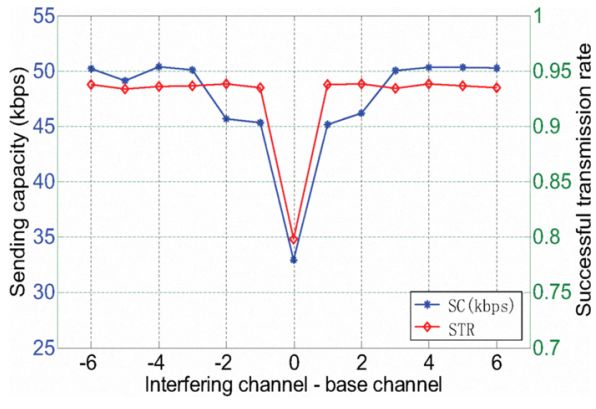

Fig. 3. Sending capacity and successful transmission rate under interfering channel

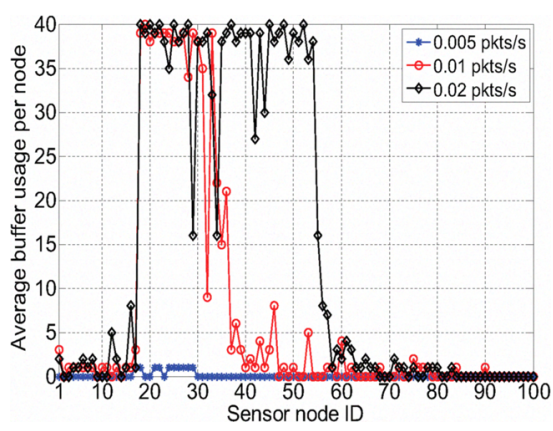

Fig. 6. Impact of data sending rate to buffer wall phenomenon presents a similar result. Moreover, we do not observe an obviously biased performance. There is no clear evidence that some channels are more preferred than others. All the channels present a similar behavior.

2) Interference among different channels: The second set of experiments focuses on the interference between different channels. In this set of experiments, two pairs of sensor nodes are put close to each other (1m away). One pair of nodes operate in a fixed base channel, whereas the other pair of nodes dynamically change their channels, from the same channel, to \pm 1 , and up to \pm 6 , to serve as the interferer. We are interested in the impact of the potential interference to sending capability and STR of the base channel as shown in Fig. 3. When the interfering channel is the same as the base channel, the interference is evident, causing $30 \%$ of STR decrease and $18 \mathrm{Kbps}$ $(35 \%)$ capacity decrease. When the difference is $1 \mathrm{MHz}( \pm 1)$ and $2 \mathrm{MHz}( \pm 2)$, the capacity decrease is $5 K b p s(10 \%)$ without notable STR decrease. When the two channels are $3 \mathrm{MHz}$ away or more, no obvious interference is observed.

3) Channel switching time and switching energy cost: We conduct two sets of experiments. One is of no channel switching and the other switches the channel for every packet transmission. $10^{4}$ packets are transmitted for each set and we approximately measure the switching time by calculating the difference between these two. The results show that the averaged switching time for a sender is about $0.34 \mathrm{~ms}$ and that for a receiver is less than that for a sender, which can ensure the successful data transmission.
In order to measure the energy consumption caused by channel switching, we record the average electric current, with and without channel switching during different transmissions. With channel switching the current is $21.4 \mathrm{~mA}$ and the transmission time is $68359 \mathrm{~ms}$ for $10^{4}$ packets. Without switching the current is $21.6 \mathrm{~mA}$ and the transmission time is $65297 \mathrm{~ms}$. With a simple calculation we can conclude that the additional energy consumption caused by channel switching is no more than $2 \%$.

4) Summary: The fast channel switching $(0.34 \mathrm{~ms})$ and the low cost (no more than $2 \%$ ) suggest a brighter future for multichannel assignment in WSNs. The switching cost will not be a major concern. Nodes are free to switch frequently if there are sufficient incentives. The control overhead, which is used to coordinate nodes to be in the same channel for communication, becomes more important. It implies that a layer-based design for channel assignment, which needs less control overhead, is more promising. The other finding is that there is no need to assign a fixed and specific channel to a communication link as channels are not discriminated. Thus, channels can be used more effectively. Another important observation is about the channel availability. Results from (a) and (b) above indicate that the number of available channels is quite large. There are 83 channels $(83 / 3=27$ orthogonal channels), which are much more than the 11 channels (three are orthogonal) in 802.11 networks. In other words, the number of channels may not be a main limitation in the design of efficient protocols. 


\section{B. The buffer wall phenomenon}

In this section we present our study on the buffer wall phenomenon. Simulation experiments are conducted to model situations of large-scale deployment in WSNs. 100 sensor nodes are deployed along a line with equal distance of $15 \mathrm{~m}$ between two adjacent nodes. They form a chain topology which has been widely used in many empirical studies (e.g., [16], [17]). An ID is assigned to each node with node 0 as the sink, node 1 as the sink's one-hop neighbor and node 100 as the most far away node to the sink. In our experiments, each node generates raw data (i.e., sensory data collected at each node) at the same fixed rate. The raw data will be transmitted to the sink using the shortest-path routing with B-MAC [18] as the MAC protocol. Thus, node $i$ has to transmit its own raw data as well as the data forwarded from node $i+1$ to node $i-1$ for $i=1$ to 99 . We further adjust the transmission power to control the interference range. When an interference range covers nodes within $k$ hops, we call it $k$-hop interference. Perfect link is assumed in the simulation. Each sensor node is allocated with a buffer to hold up to 40 regular packets. Packets that cannot be transmitted are stored in the buffer. If the buffer is full, packets would be dropped in an FIFO manner.

1) The basis of the buffer wall phenomenon: It is a common belief that nodes nearer to the sink are more likely to be congested than the ones distant to the sink (e.g., [10], [11]). This is, however, not always true according to our empirical results. In our experiments, the raw data generated at each individual sensor node will accumulatively flow towards the sink, causing the intermediate nodes to be congested rather than those nodes near to the sink. We define average buffer usage as the average amount of buffer being used after the network enters a stable state. Fig. 4 shows the average buffer usage in each individual sensor node in the chain topology with respect to three different interference ranges, under a fixed data sending rate per node of $0.02 p k t s / s$. Fig. 5 shows the goodput per node under the same configurations as in Fig. 4. Goodput for one node is defined as the number of packets received by the sink divided by the total number of packets generated by this node [8]. In the case of 16-hop interference, according to the average buffer usage, the whole network can be partitioned into three parts (as shown in Fig. 4): part A of less usage from node 1 to node 17, part B of peak usage from node 17 to node 54 , and part $C$ of less usage from node 54 to node 100 . The large interference range slows the transmission of those data. It prevents the data flows of the nodes in part $\mathrm{C}$ from congesting the nodes in part $\mathrm{A}$, while the sink can quickly consume the data in part A nodes. As a result, nodes in part $A$ have much less usage of the buffers due to the lack of data to transfer. The buffer usage of part B nodes is like a wall that blocks certain data transfer of part $C$ nodes to part A. We call this buffer wall phenomenon.

One consequence of the buffer wall phenomenon is the under-utilization of the capacities of nodes nearer to the sink (part A in this example), which are indeed the bottleneck of the

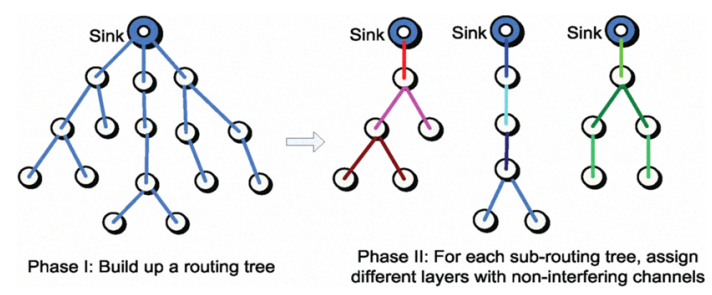

Fig. 7. Two phases in double-plate

network throughput. Their communication bandwidths are not fully utilized due to the lack of data to transfer. Consequently, the total network throughput is degraded.

The second consequence of the buffer wall phenomenon is the unfair data delivery as shown in Fig. 5. For this scenario, where nodes generate data in the same rate, if goodputs for two nodes are the same, we say the data generated by these two nodes are fairly delivered; otherwise, unfairness occurs. Also, a goodput of less than 1 for one node means some packets for the node were dropped. Fig. 5 shows that part A nodes can always achieve $100 \%$ goodput; the goodput of part B nodes decreases as the distance from the sink increases; part $\mathrm{C}$ nodes have similar goodputs but much less than 1 since their data were dropped by the buffer wall formed by part B nodes.

Furthermore, the traditional congestion control mechanisms (e.g., [8]) are based on the buffer usage to make rate adaptation. Because of the high buffer usage of the buffer wall nodes (part B in this example), those far away nodes (part C) will adapt to a lower rate. This only happens to part $C$ nodes, but not to part B or part A nodes, which leads to further unfairness.

2) Impact of interferences and data sending rates: Fig. 6 also shows the impact of different interference ranges to the average buffer usage, with a fixed data sending rate of $0.02 p k t s / s$. As the interference range grows, we observe that the buffer wall moves further away from the sink and grows wider. The second set of experiments study the impact of data sending rate, ranging from $0.005 p k t s / s$ to $0.02 p k t s / s$. The interference range is fixed as 16-hop interference. We observe that there is no change on the beginning position of the buffer wall, but the width of the buffer wall grows wider as the data sending rate increases. Also, for a fixed interference and a fixed rate, the borders of the buffer wall are fixed.

3) Summary: From these sets of experiments we can find out that the buffer usage plays a critical role in throughput and fairness improvements in WSNs. It is essential to level down the buffer wall to get better buffer usage distribution. Ideally, since nodes nearby the sink are the bottleneck of the transmission, they shall have a higher buffer usage so that their wireless bandwidth can be fully utilized. On the contrary, nodes distant to the sink shall not have the higher buffer usage which may cause a false rate adaptation.

\section{FAIR MULTI-CHANNEL ASSIGNMENT}

Section III.A implies that a layer-based channel assignment not only can reduce coordination communication overhead but also can be convenient for message broadcasting. Section III.B 


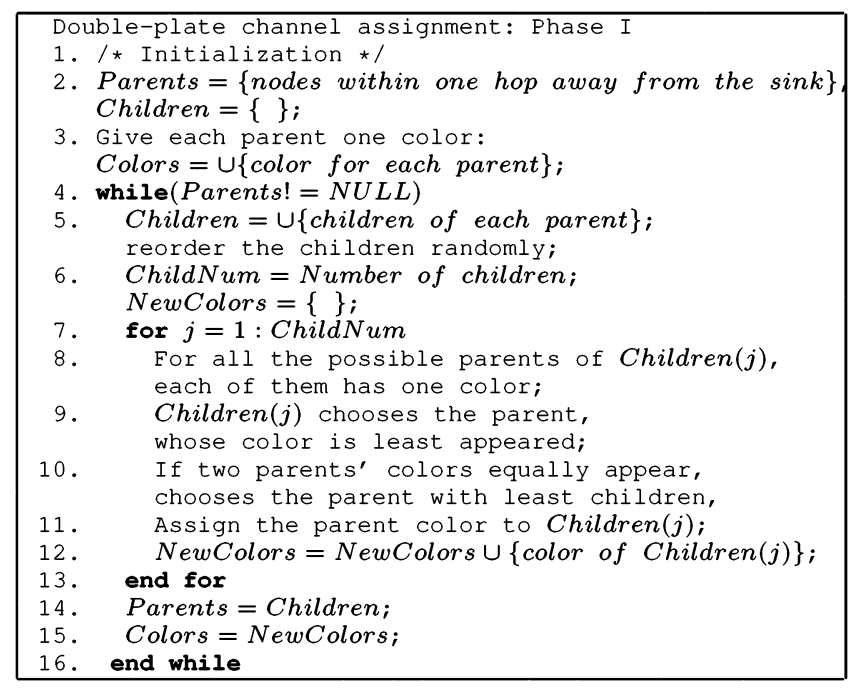

Fig. 8. Routing tree building for double-plate

indicates that in order to level down the buffer wall and to get better buffer usage distribution, each node should take nearby buffer usage distribution into consideration. These two points form the principles of our algorithm design.

We propose the double-plate channel assignment algorithm. First, each node needs to find an appropriate parent to form a routing tree. Second, for each sub-routing tree, a series of channels is assigned to it; and each node chooses a sending channel in a layer based approach. Third, each node switches channel in an asynchronous slot based manner. An intra subrouting tree channel switching scheme is proposed to make sure that nodes with higher buffer usages can get more chances to transmit. At last, nodes in one sub-routing tree may switch to another for better sending rate under an inter sub-routing tree rate adaptation scheme.

\section{A. Double-plate channel assignment}

As shown in Fig. 7, there are two phases for double-plate channel assignment. The first phase is to build a routing tree rooted at the sink. The second phase is to assign each subrouting tree with a serial of channels, each of which is assigned to one layer of nodes.

1) Phase I: routing tree building: We define a sub-routing tree as the routing tree rooted at one node that is one hop away from the sink plus the sink node itself (e.g., there are three sub-routing trees in Fig. 7 Phase I). It is required that the difference of tree topology and node number between any two sub-routing trees should be minimized. This requirement is to help promise that the optimal fair data sending rate per sub-routing tree can be the same (Section IV.D). The detailed protocol is listed as Fig. 8.

Since the above BFS based assignment can only approximate that two sub-routing trees have similar number of nodes and have similar topologies. This approximation will make the achievable data sending rate of different sub-routing trees differs from each other. Such kind of inter sub-routing tree unfairness issue will be dealt with in Section III.C.

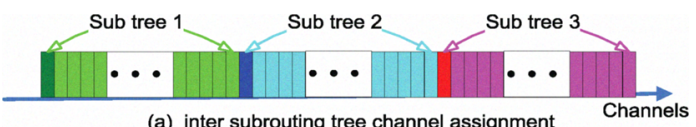

(a) inter subrouting tree channel assignment

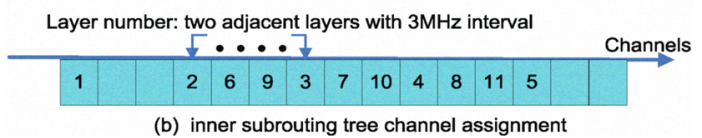

Fig. 9. Layer-based channel assignment

2) Phase II: layer-based channel assignment:

To use up the multi-channel availability effectively, a layerbased channel assignment is proposed. This assignment firstly makes sure that channels for different sub-routing trees do not interfere with each other. Then for adjacent layers in the same sub-routing tree, channel interval of no less than $3 \mathrm{MHz}$ is guaranteed.

Suppose there are $N$ available channels with two adjacent channels being $1 \mathrm{MHz}$ away, and there are $m$ sub-routing trees after Phase I. We separate the $N$ channels into $m$ sets as equally as possible as shown in Fig. 9. One set of channels is for one sub-routing tree. Then for each sub-routing tree, assign each layer a specific sending channel chosen from the assigned set of channels.

For sub-routing tree $j$, suppose there are $n$ channels assigned to it, namely channel 1 to channel $n$. Channel $n-1$ and channel $n$ are not used to make sure that the least interval of assigned channel sets for two sub-routing trees is $3 \mathrm{MHz}$ away. Let the sink be the layer 0 node. The layer for other nodes is defined as the hop number from that node to the sink. Suppose there are $K$ hops in total. Channel $(k)$ is the sending channel assigned to nodes in layer $k$. Let Channel $(1)=1$ and $k=2$. For layer $k$, if $k<=K$ :

- If Channel $(k-1)+3<n-1$, let Channel $(k)=$ Channel $(k-1)+3, k++$.

- Otherwise, if there are channels unassigned between Channel(2) and Channel $(k-1)$, assign the least used one to layer $k, k++$.

- Otherwise, if channel 1 and channels 4 to $n-2$ have all been assigned, there are two choices to continue. If layer $k$ is far away enough from layer 1 , assign Channel $(k)$ the least used channel from 2 to $n-2$; otherwise, let Channel $(k)=$ Channel $(2), k++$.

After the assignment, layer 0 node (i.e., the sink) has no interferers, ensuring that the capacity of layer 0 node can be fully utilized. Adjacent layers are assigned with channels that are $3 \mathrm{MHz}$ away. For layers that are far away to each other, channels that are less than $3 \mathrm{MHz}$ away can also be very helpful. The reason is that the $10 \%$ sending capacity decrement for two channels within $2 \mathrm{MHz}$ away happens only at the case when two links send packets with their highest rates (refer to Fig. 3), but layers further to the sink have much less traffic than layers nearer to the sink under the fair data sending rate scenario. It is quite efficient to use up the partially overlapping channels. 


\section{B. Intra sub-routing tree channel switching scheme}

Each node is assigned with one fixed sending channel as described in Section IV.A. Sending channel is used to communicate with the parent. One node should switch to its children's sending channel for receiving, which is called receiving channel for the node. An asynchronous slot based channel switching scheme is proposed to prevent coordination communication overhead between parent and children and also to achieve better buffer usage distribution.

Section III indicates that the transmission time for a regular packet is almost $7 \mathrm{~ms}$. We set one slot of time to be $21 \mathrm{~ms}$. This algorithm provides two kinds of decisions for each node, one receiving decision and one sending decision (shown in Fig. 10):

(a). receiving decision: A receiving decision contains two slots of time being in receiving channel and one slot of time being in sending channel.

(b). sending decision: A sending decision contains two slots of time being in sending channel and one slot of time staying in receiving channel.

For each node, after one period of decision time, it needs to make another decision for the next 3 slots according to the buffer usage distribution nearby the node. Since nodes in the same layer share the same sending channel, it is not hard for one node to get the buffer usage information of the nearby nodes by overhearing. Suppose node A can know the buffer usage situation of the following nodes: node A itself, node A's siblings and other peers that might interfere with A's sending, node A's children, and node A's parent. Then the decision for the next decision period can be made as following:

- If A has no children, A chooses sending decision if the buffer usage of $\mathrm{A}$ is less than its parent.

- If A has some children and A uses less buffer than either the maximal buffer usage of its children, or the sum of the buffer usages of all its children, A makes receiving decision.

- If A has some children, but A uses no more buffer than any of its children or the sum of buffer usages of its children, then A compares its buffer usage with its parent. If $\mathrm{A}$ uses more buffer than its parent, A makes sending decision; otherwise, receiving decision.

- If the buffer usages of A, A's parent, and the sum of A's children are all equal, then A compares its buffer usage with its siblings and the peers that can be overheard by A. If $\mathrm{A}$ uses more buffer than any of them, A makes sending decision; otherwise, A checks whether it has used more than half of its buffer. If yes, A makes sending decision; otherwise, A makes receiving decision.

\section{Inter sub-routing tree rate adaptation}

Inter sub-routing tree rate adaptation is proposed to keep fairness among different sub-routing trees so that the available data sending rate of one node can be dynamically increased to the height it deserves after the static channel assignment period. Since the leaf node in one sub-routing tree has no

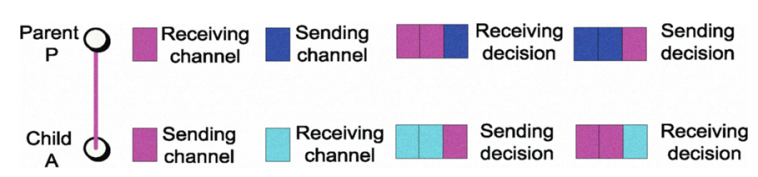

Fig. 10. Channels for each node and the corresponding sending and receiving decisions

children to listen to, the leaf node can overhear the height of fair data sending rate in another sub-routing tree by assigning its receiving channel as its potential parent's sending channel. After a period, if a node $A$ finds out that its data sending rate is stable but much lower than that of the potential parent $Q$, $A$ chooses to switch to the sub-routing tree which $Q$ belongs to.

The strategy goes like this. Since the fair data sending rate for one sub-routing tree with $\mathrm{n}$ nodes is $\frac{4}{9(2 n-1)}$ (Section IV.D), if the node number of two sub-routing trees differ by more than 2 , the leaf node may choose to switch. For node $\mathrm{A}$, if the current rate is ratei, and the rate for the potential parent is ratej, then if $\frac{4}{(9 \times \text { ratei })}-\frac{4}{(9 \times \text { ratej })}>4$, node A would choose to switch to the other sub-routing tree.

\section{Analysis}

1) Optimal fair sending rate: Suppose the layer 1 node for sub-routing tree $i$ is node $i$. Given that there are $n$ nodes in sub-routing tree $i$, the data sending rate for each individual node is $t p k t s / s$. If new packets are sending at the time when some of the previous packets are still on the way to the sink, there would be an accumulative effect that more and more packets be blocked on the way resulting in congestion. Therefore, in order to prevent the occurrence of congestion, before each node sends its new packet, all the previous $n-1$ packets should be received by node $i$, and node $i$ also needs to transmit a total of $n$ packets ( 1 for itself, and $n-1$ for the children) to the sink. Since both receiving and sending needs to consume time, node $i$ needs to work $n-1+n$ transmitting times. If the transmitting capability of node $i$ is fixed, say $B \mathrm{pkts} / \mathrm{s}$, at least $\frac{2 n-1}{B}$ seconds will be needed to forward the data. Thus, the data sending rate $t$ per node is upper bounded by $\frac{B}{2 n-1} p k t s / s$.

2) Lower bound of the proposed scheme: Under the definition of sending decision and receiving decision in Section IV.B, the overlapping time for parent receiving and child sending is no less than one slot of time in one decision period. Since the worst case is that child and parent totally missed in the first decision period. But in this way, the parent has got no packet and the child has not sent out a packet. They both choose the decision feasible for child's transmission, leading to the channel overlapping in the parent's second decision period. Thus, for each case, one link might last for at least one slot in one decision period, during which, idealistically, 3 packets can be transmitted.

For each node, when it stays in the sending channel, it firstly listens to see whether the channel is clear. If it is yes, the node sends the packet; otherwise, the node just waits until the packet 


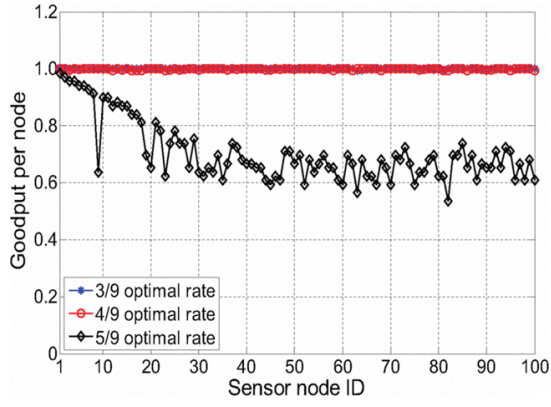

Fig. 11. Goodputs for chain topology under double-plate multi-channel assignment

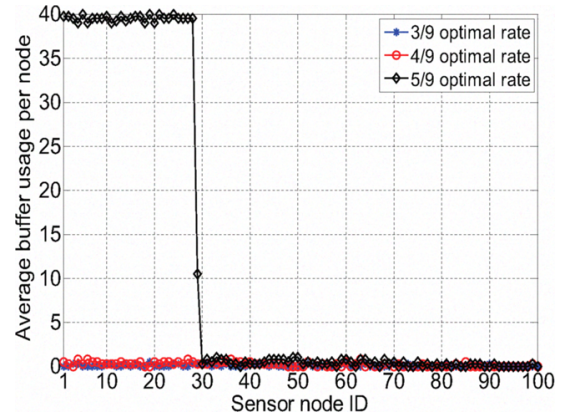

Fig. 14. Average buffer usage for chain topology under double-plate multi-channel assignment

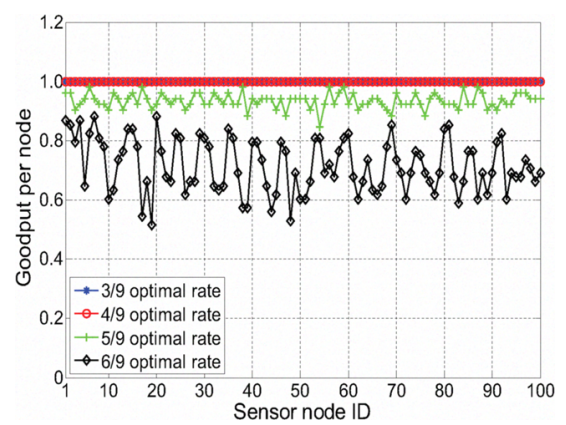

Fig. 12. Goodputs for uniformly random topology under double-plate multi-channel

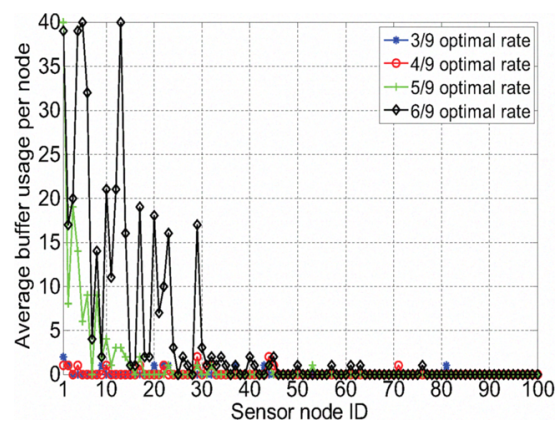

Fig. 15. Average buffer usage for uniformly random topology under double-plate multi-channel assignment

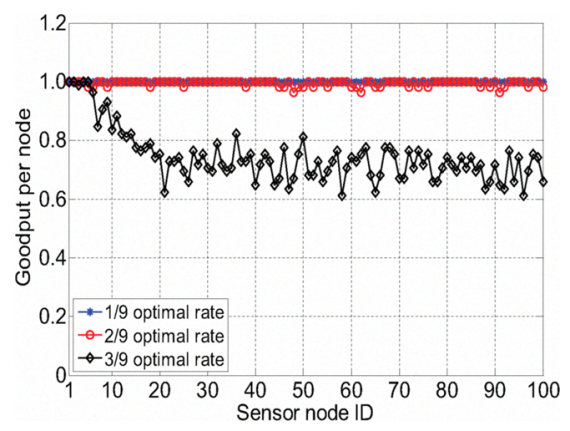

Fig. 13. Goodputs for chain topology under TMCP channel protocol

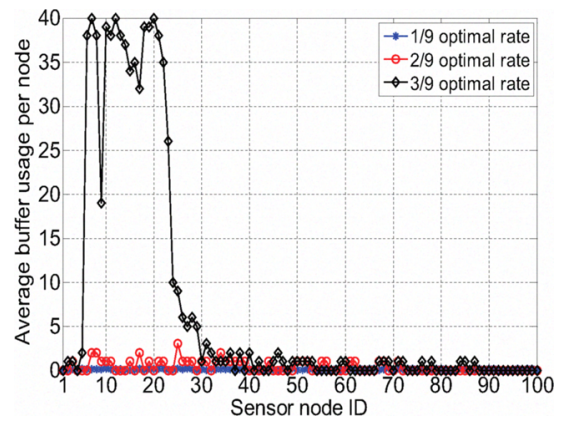

Fig. 16. Average buffer usage for chain topology under TMCP channel protocol has been sent, then sends out its own packet after a random delay. This is a purely ALOHA based approach. The well know theory about the throughput in ALOHA approach points out that the throughput decreases to no less than $18.9 \%$ [19]. Under the proposed algorithms, the chance that two nodes meet at the same time, transmitting to the same parent can be no more than $\frac{2}{3} \times \frac{2}{3}=4 / 9$. Thus, the throughput would meet a decrease of no more than $1-\left(\frac{4}{9} \times 18.9 \%+\frac{5}{9}\right)=36.04 \%$. Then, at least $3 \times(1-36.04 \%)=2$ packets can be sent on average for one decision period. For the layer 1 node of each sub-routing tree, since it has no interferers, the sending time can be fully used if there are enough packets. Optimally, one decision period can transmit $(3+6) / 2=4.5$ packets. Practically, 2 packets can be received in one decision time on average. Therefore, $2 / 4.5=4 / 9$ capacity of the layer 1 node of sub-routing tree has been used. The practical available data sending rate is $4 / 9$-approximition to the optimal result.

\section{Performance Evaluation}

Simulation and evaluation have been done to verify the performance of the proposed schemes. Because we assign noninterfering channels to different sub-routing trees, performance of one sub-routing tree is independent to another. Thus, we study the achievable data sending rate and the buffer usage distribution achieved for one sub-routing tree, and we mainly compare our work with the most recent multi-channel assignment work TMCP [6], which assigns one channel for one sub-routing tree.
We firstly define achievable fair data sending rate as following. The achievable fair data sending rate is measured using goodput defined in Section III. Suppose data sending rate generated from each individual node is the same in WSNs, if the goodput is 1 for all the nodes, which means the data from the nodes can all be fairly delivered, the data sending rate is said to be achievable. And we define optimal fair data sending rate (optimal rate) as the achievable data sending rate in the idealistic case.

We conduct a series of simulations to verify and evaluate the performance of the proposed schemes.

In the simulation part, both chain topology and uniformly random topology are used. Each topology contains $n=100$ nodes, which report their data to sink in a many-to-one manner. Node ID assignment and deployment for chain topology are the same manner as in Section III.B. For uniformly random topology, the nodes are placed in a $200 \mathrm{~m} \times 200 \mathrm{~m}$ region, with density varying from 4 to 16 . The sending capacities of the nodes are $50 \mathrm{Kbps}$. The length of a regular packet is 43bytes long. The optimal rate is thus $\frac{145}{2 n-1}$ (pkts/s) (refer to Section IV.D). The maximum buffer allocation per node is 40 regular packets. FIFO principle is used when buffer overflow occurs. Perfect links are assumed, and shortest path routing and B-MAC are implemented for data transmission. Results show that for double-plate, 4/9 optimal achievable data sending rate (referred as optimal rate in the following parts) can be achieved and the buffer usage distribution for 


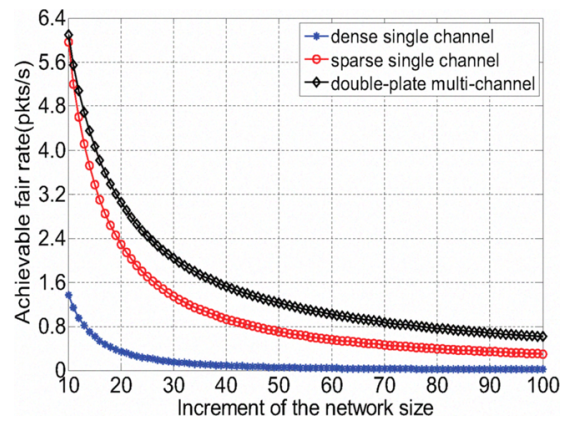

Fig. 17. Fair data sending rate comparison for different topologies

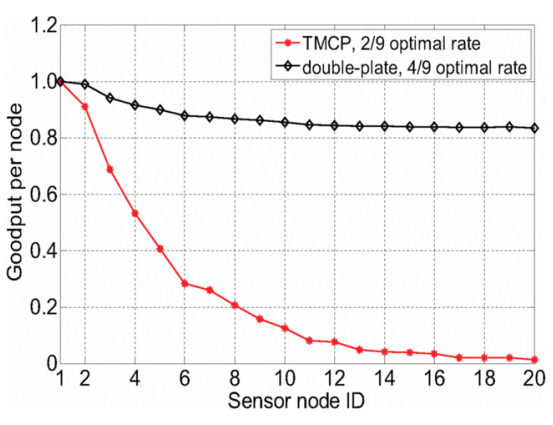

Fig. 18. Goodput per sensor node

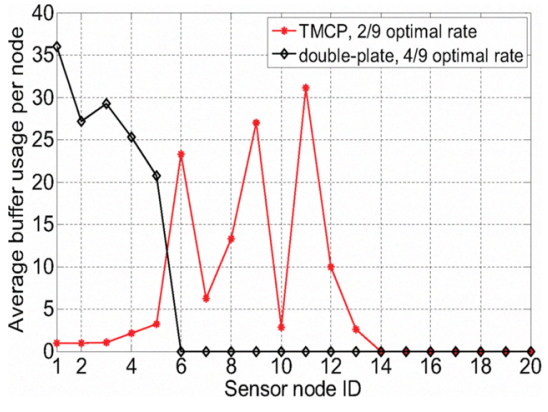

Fig. 19. Average buffer usage per sensor node the whole network can be adapted to a much better situation.

In the evaluation part, 20 Telosb sensor nodes are placed in a line, making up of a chain topology. Two adjacent nodes are placed $15 \mathrm{~m}$ away, under which the STR is almost $94 \%$. Other settings are consistent to the ones in simulation. The goodput and average buffer usage are recorded for both TMCP and double-plate protocols.

\section{A. The achievable fair data sending rate}

Both chain and uniformly random topology with 100 nodes are tested. There are 10 available channels for each topology with 4 hop interference. Thus, the double-plate assignment can promise a layer interference-free feature.

In Fig. 11 with the chain topology, a data sending rate of $3 / 9$ optimal rate to $5 / 9$ optimal rate are tested. It is clear that a rate of no more than $4 / 9$ optimal rate is achievable. For $5 / 9$ optimal rate, the goodputs for the nodes further to the sink are decreased, but still in a quite fair manner. Fig. 12 studies the uniformly random topology case, with data sending rate changing from $3 / 9$ to $6 / 9$ optimal rate. Not only the $4 / 9$ optimal rate can be achieved, all the nodes can achieve $90 \%$ goodputs under $5 / 9$ optimal rate. The reason is that, with more nodes in the same layer comparing to the case in the chain topology, the receiving period for one parent node can be better utilized (as described in Section IV.D). Thus, the capacity of the layer 1 node, which is nearest to the sink, can be better utilized. Fig. 13 depicts the TMCP chain case. It can be seen from the figure that at most a rate of $2 / 9$ optimal rate can be achieved. When the rate grows higher, the unfairness becomes larger for nodes further away to the sink, while the nodes nearer to the sink can always get $100 \%$ goodputs. The next part will explain this by viewing the buffer usage distribution for each case.

\section{B. Average buffer usage distribution}

This section describes the buffer usages for the three cases studied in Section V.A. Fig. 14 is for chain topology under double-plate. All the buffers are almost empty for $3 / 9$ and $4 / 9$ optimal rate cases. When the rate grows up to $5 / 9$ optimal rate, there are lots of data stored in the first 30 nodes which are nearer to the sink. This shows that the $5 / 9$ optimal rate is out of the capacity of the proposed scheme. But the average buffer usages for the first 30 nodes are still quite fair due to the channel switching scheme proposed in Section IV.B. Also, the nodes further to the sink does not use more buffer than the nodes nearer to the sink, resulting in a better utilization of the capacities of those bottleneck nodes.

Fig. 15 shows similar results for the uniformly random topology. When data sending rate is not bigger than $4 / 9$ optimal rate, all buffers are almost empty. When the rate grows to $5 / 9$ or even $6 / 9$ optimal rate, the buffer usages for the nodes nearer to the sink start to increase.

On the contrary, the TMCP case as depicted in Fig. 16 shows a clear buffer wall in the middle, which results in unfair goodputs for nodes further to the sink. Also, nodes nearer to the sink can always achieve $100 \%$ goodputs, which differs from cases in double-plate. Nodes nearer to the sink in doubleplate cannot all achieve $100 \%$ goodputs once the sending rate exceeds the capability. That is because the parent node always uses more buffer than the children in double-plate. The data for children has to wait in the parent's buffer for transmission. Once the buffer is full for one parent, the data for the children are dropped in an equal manner, causing the decrement of the goodputs in a fair manner for all the children.

\section{Achievable rate comparison and interference effect}

Let $n$ be the number of nodes in a WSN and $B(p k t s / s)$ be the sending capacity of each sensor node. The achievable data sending rate is at most $O\left(\frac{B}{n \log n}\right)$ [8] for a sparse balanced tree in the single channel case. For other topologies, like unbalanced or dense trees, the capacity is almost $O\left(\frac{B}{n^{2}}\right)$ [8]. For double-plate, the achievable data sending rate is $\frac{4 B}{9 n}(p k t s / s)$. Fig. 17 shows that when $n>10$, the doubleplate is always better than the other two cases.

When $n$ grows larger, the difference becomes bigger. If $n=$ 100 , we have $\frac{4 / 9 n}{1 / n \log n}=2$. This means that double-plate can handle two times of the data generation rate comparing with the case when one sub-routing tree has only one channel like TMCP. If the interference range is larger, the difference of achievable data sending rate between double-plate and TMCP grows to $\frac{4 / 9 n}{1 / n^{2}}=\frac{4 n}{9}$, a potential for significant improvement.

Section III.B describes the buffer wall phenomenon indicating that a single channel tree suffers from a bigger buffer wall with a bigger interference range, leading to the 
greater unfairness among the trees. The proposed doubleplate shows better robustness in fairness when the interference range is increased comparing with the case of TMCP because double-plate assigns two adjacent layers with non-interfering channels. This will dilute the interference effect and the consideration of nearby buffer usage distribution for one node can also help further level down the buffer wall.

\section{Evaluation on Telosb test-bed}

20 Telobs nodes, putting in a chain topology, are tested with the data sending rate per individual node of $4 / 9$ optimal Rate. Both TMCP and double-plate protocols are tested. Fig. 18 studies the goodput per node and Fig. 19 depicts the average buffer usage distribution. The evaluation results are consistent with the ones obtained in simulation. The goodput per node for double-plate is fair and much higher than that of TMCP. The buffer usage for double-plate does not form a buffer wall. There are two major differences between simulation and real measurements. First, the goodputs under double-plate are not 1 for all the nodes. There is almost $20 \%$ decrease for the nodes further to the sink. This is due to the imperfect transmission link in practice. Second, there is a seemingly imperfect buffer usage distribution for node 2 in double-plate, which is a little lower than node 3 . By measuring the sending capacity, the reason is revealed that node 2 always has a slightly higher sending capacity than node 3 due to hardware factors.

\section{Conclusions}

Multi-channel capability brings us new opportunities for data transmissions in WSNs. Traditional algorithms address the multi-channel assignment issue and the fairness control in an isolated manner, without joint considerations for the tradeoff in between. Noticing this we conducted a large number of empirical studies. One of the major observations is that in WSNs, the number of orthogonal channels is quite large. Further, the channel switching time and the switching energy cost are relatively small. It implies that frequent channel switching in a packet granularity is feasible and promising in certain circumstances. The second observation is the buffer wall phenomenon which is one of the main reasons for the degraded network throughput and node unfairness. To address the problem, we proposed a novel layer-based multi-channel assignment algorithm. The key idea of the proposed algorithm is to level down the buffer wall so that a better trade-off between the network throughput and fairness can be achieved with a maximized minimal achievable data sending rate from individual nodes. We proved that our proposed algorithm can guarantee $4 / 9$ of the optimal data sending rate in the worst case. The experimental results showed that the achievable data sending rate can get an improvement of up to $100 \%$ comparing with the existing work TMCP.

The future work can be carried out along following directions. First, in our network model we assume that wireless links are perfect with $100 \%$ delivery ratio. In practice, however, wireless links are lossy and the qualities of links are dynamically changing. How to solve the problem in a real environment is one of the main challenges. Second, we consider only the many-to-one communication pattern. There are, however, some other useful patterns, which may raise more challenges. Finally, we consider a static WSN with a single sink. In many applications, sensor nodes may have mobility and there may have a number of sinks. All these issues are left open for future research.

\section{ACKNOWLEDGEMENTS}

This research was supported in part by the Hong Kong RGC grant HKUST617908, the National Basic Research Program of China (973 Program) under Grant No. 2006CB303000, and HKUST Nansha Research Fund NRC06/07.EG01.

\section{REFERENCES}

[1] I. F. Akyildiz, W. Su, Y. Sankarasubramaniam and E. Cayirci, "A Survey on Sensor Networks", in IEEE Communications Magazine, 2002, Vol.40:102-114.

[2] S. Kim, S. Pakzad, D. Culler, J. Demmel, G. Fenves, S. Glaser and M. Turon, "Health Monitoring of Civil Infrastructures Using Wireless Sensor Networks", in IEEE IPSN, 2007.

[3] S. Motegi, K. Yoshihara and H. Horiuchi, "Implementation and Evaluation of On-demand Address Allocation for Event-Driven Sensor Network", in SAINT, 2005.

[4] W. Wang, Y. Wang, X. Li, W. Song and O. Frieder, "Efficient Interference-Aware TDMA Link Scheduling for Static Wireless Networks", in ACM MobiCom, 2006.

[5] P. Kyasanur and N. H. Vaidya, "Capacity of Multi-channel Wireless Networks: Impact of Number of Channels and Interfaces", in ACM MobiCom, 2005.

[6] Y. Wu, J. A. Stankovic and T. He, "Realistic and Efficient MultiChannel Communications in Dense Wireless Sensor Networks", in IEEE InfoCom, 2008.

[7] G. Zhou, C. Huang, T. Yan, T. He, J. A. Stankovic and T. F. Abdelzaher, "MMSN: Multi-Frequency Media Access Control for Wireless Sensor Networks", in IEEE InfoCom, 2006.

[8] S. Rangwala, R. Gummadi, R. Govindan and K. Psounis, "InterferenceAware Fair Rate Control in Wireless Sensor Networks", in ACM SigComm, 2006.

[9] J. Paek and R. Govindan, "RCRT: Rate-Controlled Reliable Transport for Wireless Sensor Networks", in ACM SenSys, 2007.

[10] G. Ahn, E. Miluzzo and A. T. Campbell, "Demo Abstract: A FunnelingMAC for High Performance Data Collection in Sensor Networks", in ACM SenSys, 2006.

[11] H. Gupta, V. Navda, S. Das and V. Chowdhary, "Efficient Gathering of Correlated Data in Sensor Networks", in ACM MobiHoc, 2005.

[12] P. Bahl, R. Chandra and J. Dunagan, "SSCH: Slotted Seeded Channel Hopping for Capacity Improvement in IEEE 802.11 Ad-Hoc Wireless Networks", in ACM MobiCom, 2004.

[13] J. Zhang, G. Zhou, C. Huang, S. H. Son and J. A. Stankovic, "TMMAC: An Energy Efficient Multi-Channel MAC Protocol for Ad Hoc Networks", in ICC, 2007.

[14] [Online]. Available: http://www.tinyos.net/tinyos-2.x/doc/html/tep111.html.

[15] Texas Instruments, CC2420: $2.4 \mathrm{GHz}$ IEEE 802.15.4/ZigBee-ready RF Transceiver Datasheet. http://focus.ti.com/lit/ds/symlink/cc2420.pdf.

[16] Q. Cao, T. he, L. Fang, T. Abdelzaber and J. S. Son, "Efficiency Centric Communication Model for Wireless Sensor Networks", in IEEE InfoCom, 2006.

[17] S. Lin, J. Zhang, G. Zhou, L. Gu, T. He and J. Stankovic, "ATPC: Adaptive Transmission Power Control for Wireless Sensor Networks", in ACM SenSys, 2006.

[18] J. Polastre, J. Hill and D. Culler, "Versatile Low Power Media Access for Wireless Sensor Networks", in ACM SenSys, 2004.

[19] F. F. Kuo, "The ALOHA system", in ACM Computer Communication Review, 25(1), 1995. 